\title{
marges Marges
}

revue d'art contemporain Revue d'art contemporain

\section{Quand l'image condense le temps. Visite} extraordinaire au Musée de l'Ermitage dans

\section{L'Arche russe}

When the image condenses time. An extraordinary visit to the Hermitage

Museum in The Russian

\section{Francis Gauvin}

\section{OpenEdition}

Journals

Édition électronique

URL : http://journals.openedition.org/marges/941

DOI : $10.4000 /$ marges.941

ISSN : 2416-8742

\section{Éditeur}

Presses universitaires de Vincennes

Édition imprimée

Date de publication : 1 octobre 2014

Pagination : 26-37

ISBN : 978-2-84292-415-7

ISSN : $1767-7114$

\section{Référence électronique}

Francis Gauvin, «Quand l'image condense le temps. Visite extraordinaire au Musée de l'Ermitage dans L'Arche russe », Marges [En ligne], 19 | 2014, mis en ligne le 01 octobre 2016, consulté le 19 avril 2019. URL : http://journals.openedition.org/marges/941; DOI : 10.4000/marges.941 


\section{Quand l'image condense}

le temps. Visite extraordinaire au Musée de l'Ermitage dans L'Arche russe.

/1 Martin Heidegger, Le Principe de raison, trad. A. Préau, Paris, Gallimard, 1962, p. 183.

/2 Alain Fleischer, Les Laboratoires du temps.

Écrits sur le cinéma et la photographie, tome I, Paris, Galaade Éditions, 2008, p. 344.

/3 ibid.

\section{«Ce qui vient à nous est en route depuis longtemps/1. »}

Dans son livre intitulé Les Laboratoires du temps, Alain Fleischer énonce qu'un «musée n'est pas plus conçu pour être filmé qu'une architecture n'est imaginée pour donner de belles ruines/2 ». Pour lui, le musée ne peut qu'être expérimenté: « Il est d'abord destiné au visiteur [...] qui reste libre de son parcours : hésitations, explorations, haltes prolongées, accélérations, ralentissements, retours en arrière, raccourcis, évitements. Le visiteur à qui le musée est destiné n'est pas le passager d'un de ces trains fantômes des fêtes foraines dont l'itinéraire est obligatoirement ponctué par des spectacles et des surprises qui attendent à poste fixe. Ces chariots, ces rails, ces mouvements réglés, ces perspectives organisées sont ceux du film, de la caméra/3. ". Certes, un musée doit être expérimenté de l'intérieur. Une visite externe, par le truchement d'une caméra, ne rend pas justice à l'expérience muséale, ni au musée en soi. Cela dit, et sans rien vouloir enlever à la pertinence de cette remarque, lorsqu'Alexandre Sokourov

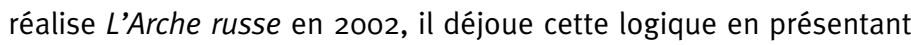
une expérience filmique qui manifeste quelque chose d'essentiel à la déambulation muséale. En tant qu'arche de la culture, le musée exerce une tension entre le passé et le présent, et cette tension est au cœur de son film. Ce dernier donne à voir l'espace comme la trace vivante d'un héritage culturel.

Véritable tour de force cinématographique, non seulement des points de vue technique et esthétique, ce film qui fut tourné en un seul plan-séquence, et qui met en scène pas moins de trois orchestres, près de 900 acteurs et figurants, propose un voyage à 
travers l'histoire du musée le plus important de Russie : l'Ermitage. De fait, la caméra parcourt les différentes époques appartenant à l'histoire du musée comme si elle était dans un lieu de contemporanéité où tous les temps sont présents en même temps. Les divers évènements historiques se chevauchent dans un fil continu à travers l'image, sans toutefois que leur ordre chronologique soit respecté. Le film opère des sauts temporels qui court-circuitent la linéarité du temps, et permet ainsi de juxtaposer l'histoire du Musée de l'Ermitage à même son récit, exactement comme le musée juxtapose l'histoire de l'art entre ses murs.

L'Arche russe est un film d'une grande complexité, et il pourrait être étudié sous plusieurs angles. L'analyse pourrait s'intéresser d'une part au discours que tient le narrateur tandis qu'il commente sa visite dans le musée de l'Ermitage, afin de comprendre son interaction avec l'image, comment l'un et l'autre donnent sens au film. D'autre part, on pourrait se pencher plus précisément sur le travail de réalisation qu'effectue Sokourov alors qu'il tourne ce film en un seul plan-séquence, en interrogeant le sens qu'on peut attribuer à cette pratique au regard de la volonté du réalisateur. Ce n'est cependant ni à l'intérieur de la diégèse, comme le propose la première piste de lecture, ni selon ses conditions de production, comme pourrait le suggérer la seconde, que cet article entend approcher L'Arche russe, mais dans une position intermédiaire, c'est-à-dire entre le regard de la caméra et l'image qu'il donne à voir. L'hypothèse défendue est que la tension temporelle qu'on retrouve dans L'Arche russe s'exerce précisément là, entre un donnant à voir et un donné à voir.

Pour ce faire, il faut porter une attention toute particulière à l'image cinématographique afin de relever les indices plastiques à partir desquels cette tension est mise en œuvre. Catherine Saouter rappelle à cet effet que « la sémiose visuelle exige de faire clairement la distinction entre le donnant à voir et le donné à voir/4. ». Le donné à voir, c'est l'iconicité de l'objet, ce qu'on reconnait à travers une représentation, tandis que le donnant à voir, c'est sa construction formelle, sa plasticité. Or, s'il importe de faire la distinction entre ces deux plans, c'est pour éviter de s'emporter dans des considérations esthétiques ou idéologiques qui risquent de nous faire perdre de vue l'œuvre étudiée. Le film de Sokourov prescrit d'ailleurs de porter une telle attention. C'est du moins ce dont témoigne la scène où le Marquis de Custine rencontre, pour servir la logique avancée, une aveugle. Suite à un bref échange où elle lui fait part de ses nombreuses connaissances en histoire de l'art et en muséographie, cette femme demande au Marquis de l'accompagner jusqu'à la toile Le Festin chez Simon le Pharisien de Rubens. Arrivé avec elle dans la salle où le tableau est
/4 Catherine Saouter, Le Langage visuel, Montréal, XYZ, 2000, p. 89. 
/5 Andreï Tarkovski, «De la figure cinématographique », Positif, $\mathrm{n}^{\circ} 249$, décembre 1981, p. 35 . exposé, Custine hésite comme s'il n'y figurait plus, après quoi il feint de le retrouver. Tout le génie de la mise en scène relève alors de la disposition des personnages, car lorsque le Marquis conduit la femme devant le tableau, il la fait pivoter de manière à ce qu'elle lui tourne le dos. Ainsi quand il lui annonce qu'elle se trouve devant la toile, le subterfuge se dévoile alors qu'elle s'extasie devant le vide. Au-delà de la nature humoristique de cette séquence, il y a bel et bien une volonté d'illustrer l'importance de l'analyse plastique, sans quoi on risque d'être frappé de cécité face à toute œuvre. Et dans le cas présent, c'est au cœur même de l'image cinématographique, dans sa double articulation entre un donnant à voir et un donné à voir, que l'analyse entend se déployer.

\section{L'histoire juxtaposée}

En partant de l'idée que l'image cinématographique peut exercer une "pression du temps », il est possible de comprendre en quoi la représentation du temps est problématique dans l'œuvre de Sokourov. Dans un article de la revue Positif, Andrei Tarkovski écrit que « la consistance du temps qui s'écoule dans un plan [cinématographique], sa tension ou, au contraire, sa "raréfaction" peut être appelée pression du temps dans le plan/5 ". Cependant, la pression temporelle n'est pas seulement de l'ordre d'une tension ou d'une raréfaction, mais plutôt de l'ordre d'une juxtaposition, voire d'une condensation. Autrement dit, plusieurs temps se rencontrent dans le même plan pour ne donner à voir qu'un seul et même temps: celui de l'image.

Ce qui pose problème dans L'Arche russe, c'est que le plan-séquence donne à voir, en une seule trame continue, plus de deux cents ans d'histoire. II n'y a rien de nouveau quant au fait qu'un film condense en quelques heures un grand laps de temps. Pensons par exemple à la production canadienne Le Violon rouge (1988), de François Girard, où le récit relate l'histoire d'un violon qui se promène de mains en mains, depuis sa fabrication au XVIII' siècle jusqu'à sa vente aux enchères à l'époque contemporaine; ou à tout autre film qui intègre des analepses, soit des flashbacks, au sein de sa trame narrative afin d'éclairer ou changer la perspective qu'on peut avoir sur le récit premier.

À la différence de ces films, la grande innovation de L'Arche russe est qu'il opère des transitions temporelles à l'intérieur d'un même planséquence, ce qui désamorce en quelque sorte l'idée d'anachronisme. Comme la trame narrative se déploie en un seul souffle, le film inscrit plutôt l'entièreté de l'histoire du musée à même son cadre, et ce, sans ellipse. Et même si on voulait croire que des ellipses se dissimulent 
à travers la mise en scène, la présence de la voix off contredit cette hypothèse puisqu'elle assure la continuité narrative. De fait, dès les premiers instants du film on comprend qu'on a affaire à une caméra subjective parce que le narrateur se repère en fonction de ce qu'il voit à travers l'image cinématographique. Il faut donc admettre que le long travelling correspond à la promenade d'un seul personnage: celle du narrateur qui est représenté par la caméra subjective et la voix off qui nous livre ses pensées.

Aussitôt que la caméra entre dans le musée, sa déambulation n'est pas seulement physique ou spatiale, elle est également temporelle. Grâce à sa mise en scène, le film reproduit de nombreuses scènes historiques, qui sont toutes évidemment liées à l'histoire du Musée de l'Ermitage. À travers ces scènes, le narrateur croise plusieurs figures historiques, qui apparaissent toutes comme autant de spectres qui hantent le musée. On reconnait par exemple Pierre le Grand, qui est à l'origine de la construction du château, Catherine II, l'impératrice qui a fait l'acquisition de nombreuses collections d'art et qui, par làmême, a fondé la collection du musée, ainsi que le Marquis de Custine, un aristocrate français qui a souvent visité le château, et qui est devenu célèbre grâce à la publication de l'ouvrage La Russie en 1839. Parmi les évènements qui témoignent de la grande amplitude historique couverte par ce film, on peut penser à la scène où le Marquis, ouvrant une porte au hasard, se trouve alors en plein cœur de la Seconde Guerre mondiale. Il entre dans une pièce glacée où toutes les fenêtres ont été brisées par les bombardements, et à l'intérieur de laquelle, sous une nuée de flocons de neige, on aperçoit un homme s'affairant à emballer des œuvres d'art. Surpris par cette présence, l'homme cesse son travail et explique au Marquis qu'ici il n'y a personne, seulement des cadavres et des cercueils (en faisant référence aux toiles ainsi qu'à leur cadre).

Dans une conférence qu'il a donnée récemment/6, Alain Fleischer expliquait que l'histoire est quelque chose de feuilleté. Ce qui est passé n'est jamais définitif: il ne peut que sortir du champ de vision et, en changeant de perspective, on peut découvrir à nouveaux frais ce qui fut oublié. Le film de Sokourov s'accorde avec cette idée, car l'histoire du musée semble être emprisonnée entre ses murs. De nombreuses couches historiques s'y juxtaposent et, selon la perspective dans laquelle on se trouve, il est possible de sauter d'une époque à l'autre. Dans la scène où Catherine II assiste à la répétition d'une pièce de théâtre par exemple, on peut voir la manière dont le mouvement du regard objective ingénieusement l'opération des sauts temporels. Bien qu'il soit difficile de rendre à l'écrit la subtilité avec laquelle la perception glisse d'une vue en plongée montrant l'orchestre dans sa
16 La conférence a eu lieu le 14 mars 2013 à l'Université du Québec à Montréal, alors qu'Alain Fleischer était invité au cours d'un séminaire de Pierre Ouellet. 
/7 Gérard Genette, Figures III, Paris, Seuil, 1972, p. 78-89.

fosse à un plan large sur l'action de la scène - tandis que les acteurs dansent sur un fond brillant de pyrotechnies -, avant de s'en éloigner pour montrer en gros plan Catherine II debout au balcon; on comprend que ce glissement d'une perspective à l'autre dans l'espace, toujours dans le plan-séquence, permet de traverser les couches feuilletées de l'histoire.

Lorsque Catherine II entre dans le cadre de l'image, et qu'on aperçoit toujours la pièce de théâtre à l'arrière-plan, le changement de focalisation ne modifie pas seulement la perspective, mais entraine également un écart historique. Comme la focalisation est d'abord mise sur la pièce de théâtre, on est plongé - ne serait-ce que brièvement - dans sa diégèse et, dès que la caméra poursuit son déplacement pour encadrer Catherine II, la focalisation se déplace alors sur sa réaction avec l'auditoire. Du coup, ce n'est plus la même histoire qui est mise en scène. Et il m'importe peu de distinguer ici l'histoire fictive de l'histoire réelle: ce qu'il faut remarquer c'est le glissement de perspective qui permet de passer d'une histoire à l'autre (qu'elles soient fictives ou non), car les divers évènements qui appartiennent à l'histoire du Musée de l'Ermitage se relaient de la même manière dans le film, précisément comme s'ils étaient tous juxtaposés les uns à côté des autres et qu'en fonction du mouvement de la caméra ils pouvaient entrer et sortir de son cadre.

C'est pour cette raison qu'il est difficile de parler d'anachronisme. Dans Figures III, Genette exprime clairement que l'anachronisme concerne l'ordre dans lequel les évènements sont présentés. C'est-àdire qu'il a à voir avec leur succession/7. Or, comme le film est tourné en un seul plan-séquence, et que son action se déroule dans un seul et même souffle, il n'y a qu'un récit, et c'est celui d'un narrateur qui parcourt l'histoire du Musée de l'Ermitage. Même si les divers évènements historiques auxquels il est exposé n'appartiennent pas à la même temporalité, ils s'inscrivent néanmoins à l'intérieur de sa promenade qui, quant à elle, est tout ce qu'il y a de plus linéaire.

\section{Le panorama historique}

S'il y a une tension temporelle dans L'Arche russe, c'est parce que le temps se dédouble, entre d'un côté, le présent du narrateur et de l'autre, le passé auquel il est confronté. Ainsi, quand on considère l'amplitude historique qui est donnée à voir par l'image cinématographique, on serait porté à croire que le temps est élastique, puisque c'est comme si toute l'histoire du musée était condensée à l'intérieur du champ de la caméra, et que celle-ci n'avait qu'à ajuster sa lentille pour focaliser sur une époque précise. Ce faisant, l'image acquiert une 
profondeur de champ accrue et donne l'impression de superposer différentes couches de temps de manière à offrir un panorama historique. Employer ici le terme de "panorama », c'est à défaut d'avoir une terminologie plus appropriée pour rendre compte du phénomène en question. La notion doit être pensée de manière temporelle, c'està-dire qu'au lieu de dénoter une vaste étendue d'espace perçue à partir d'un seul point de vue, elle représente, dans ce cas-ci, une vaste étendue historique. Et si l'on voulait objecter qu'il s'agit d'un simple survol historique, comme c'est le cas par exemple dans 2001, l'Odyssée de l'espace de Stanley Kubrick où des séquences de la préhistoire précèdent des séquences futuristes, les choses ne sont pas aussi simples dans L'Arche russe, précisément à cause de l'absence de montage ou de césure.

Pour illustrer la logique qui est à l'œuvre, un indice sonore dans la séquence d'ouverture mérite d'être évoqué: ce sont les rires féminins qu'on peut entendre avant même que le narrateur n'ouvre les yeux et qu'il n'aperçoive le carrosse avec ses passagères. Comme ces rires sont difficilement audibles, comparativement à la voix off, une profondeur de champ s'instaure d'emblée entre la proximité de cette voix et l'éloignement des rires. Mais cette profondeur de champ n'indique pas seulement la distance physique entre ces deux voix: elle témoigne également de la tension temporelle qui traverse et organise L'Arche russe. C'est à l'instar des rires qui proviennent de loin que l'histoire du musée, tel un écho du passé, entre en relation avec quelque chose de proximal, soit la perception, au présent, du regard de la caméra tandis qu'elle parcourt le musée.

À ce compte, l'élément le plus important à considérer est que la caméra subjective ne se contente pas de saisir ou témoigner de l'histoire du musée, comme s'il s'agissait d'un documentaire ou d'un survol historique. Elle interagit avec l'histoire à laquelle elle est confrontée et devient par là-même tout à la fois acteur et spectateur. Phénoménologiquement parlant, on pourrait dire que son travelling, ainsi que son jeu de focalisation simulent l'action de notre propre regard tandis que nous nous déplaçons dans le monde. Elle reproduit en ce sens une errance qui ne va pas sans rappeler celle du narrateur d’ $A$ la recherche du temps perdu, où tout ce qu'il observe est susceptible d'éveiller des échos du passé, des souvenirs enfouis (pensons à la madeleine), des bribes d'histoire, etc.

À la manière d'un regard vécu, incarné, la caméra ne se pose jamais, elle n'est jamais entièrement stationnaire, sauf aux tout derniers instants du film où l'image fixe l'horizon: moment où le narrateur dit avoir découvert l'éternité. Dans cette troublante séquence qui évoque évidemment la mort, on est en droit de se demander, sémiotiquement, 
/8 Si on voulait être plus précis, on pourrait parler de « cyclorama », car contrairement au panorama, il ne peut pas être perçu en entier, d'un seul coup d'œil: il y a toujours des zones qui échappent au regard. Mais pour les fins de la présentation, les modalités du panorama suffisent.

/9 « C'est que le cinéma, encore plus directement que la peinture, donne un relief dans le temps, une perspective dans le temps: il exprime le temps lui-même comme perspective ou relief. ". Gilles Deleuze, L'image-mouvement. Cinéma 1, Paris, Minuit, 1983 , p. 39.

/10 Gilles Deleuze, L'image-temps. Cinéma 2, Paris, Minuit, 1985, p. 106.

/11 ibid., p. 112. ce que le narrateur entend par «éternité ». De fait, qu'y a-t-il d'éternel dans ce plan, sinon la présence de l'image qui persiste dans son instantanéité ? C'est-à-dire l'instant même qui est voué à être éternellement capté par un regard. Regard qui, indépendamment de ce qui est donné à voir, agit en tant que cadre de la perception. C'est lorsque l'on se demande ce que l'on aperçoit à l'intérieur de ce cadre, soit à même l'image, que des processus sémiosiques sont activés, et ceuxci font apparaître - tels des spectres - des figures du passé. À l'instar de l'épais brouillard qui hante le plan final, les multiples couches de l'histoire envahissent le Musée de l'Ermitage, donnant ainsi raison au fait que le musée soit rempli de cadavres et de cercueils.

\section{Perspectives sur le temps}

Il est possible d'approfondir l'analyse à partir de la notion de « panorama/8 ", car celle-ci permet de distinguer un point de perspective, en l'occurrence le regard du narrateur, de la vaste étendue qui se juxtapose sous ses yeux. En ce qui concerne L'Arche russe, on a vu que cette étendue est temporelle, qu'elle correspond en fait à l'histoire du musée à l'intérieur duquel le narrateur déambule, et c'est précisément dans la relation entre ce point de perspective et l'histoire à laquelle il est confronté que se joue la problématique du temps.

L'Arche russe donne raison à Gilles Deleuze lorsqu'il écrit que le cinéma est un médium privilégié pour offrir une perspective sur le temps/9, puisqu'il rend palpable la rencontre entre un présent et son passé. Comme l'explique Deleuze, l'image au cinéma se divise selon l'image actuelle, qui correspond à l'instant - éternel présent - capté par la caméra, et l'image virtuelle qui est - dans ce cas-ci - l'histoire apparaissant au sein de l'image: "Le présent, c'est l'image actuelle, et son passé contemporain, c'est l'image virtuelle, l'image en miroir/10. »; « [...] L'image actuelle et l'image virtuelle coexistent et cristallisent, elles entrent dans un circuit qui nous ramène constamment de l'une à l'autre, elles forment une seule et même "scène" où les personnages appartiennent au réel et pourtant jouent un rôle/11. ». Outre le fait d'avoir tourné ce film en un seul plan-séquence, le coup de génie de Sokourov est d'avoir réalisé L'Arche russe à travers la perspective d'une caméra subjective. Comme la caméra subjective instruit un présent sur le plan de l'énonciation, ce procédé stylistique met en évidence le contraste qui s'opère entre le cadre de l'image, toujours au présent, et l'histoire qui s'actualise dans l'image.

Ce faisant, son film rejoint d'autant plus l'idée deleuzienne que c'est dans la rencontre de l'image actuelle et de l'image virtuelle que les personnages appartiennent au réel et pourtant jouent un rôle, 
puisque le cadre de l'image correspond en fait à un regard vécu, celui du narrateur, et que celui-ci est homodiégétique, c'est-à-dire qu'il participe au récit qu'il donne à voir en focalisation interne. On retrouve ainsi en filigrane l'idée développée plus tôt voulant que le narrateur de L'Arche russe est tout à la fois acteur et spectateur de l'histoire au sein de laquelle il se trouve. Le film ne raconte donc pas simplement les péripéties d'un personnage qui voyage dans le temps, mais bien celles d'un regard qui est confronté à l'histoire qui - en le précédant - préfigure son temps.

Cela dit, quel est son temps? Il serait impossible de déterminer à quelle époque le narrateur appartient car, même si on peut estimer qu'il nous est contemporain, le film ne donne pas d'indice à cet effet. Serait-ce pertinent de savoir s'il appartient à une époque précise? L'intérêt du film est plutôt de mettre en scène le mouvement même par lequel un élément présent entre en relation avec le passé qui l'enracine. En considérant ainsi les choses, le film poétise la dialectique historique qui, à en croire Walter Benjamin, s'énonce à même l'image : "Chaque présent est déterminé par les images qui sont synchrones avec lui [...]. Il ne faut pas dire que le passé éclaire le présent ou le présent éclaire le passé. Une image, au contraire, est ce en quoi l'Autrefois rencontre le Maintenant dans un éclair pour former une constellation. En d'autres termes: l'image est la dialectique à l'arrêt/12. ».

C'est donc dire que c'est dans l'image que la rencontre entre le présent et le passé s'opère. En ce sens, si des images peuvent être synchrones avec le présent, c'est parce qu'elles ne lui appartiennent pas: elles ne sont pas actuelles. Elles appartiennent au regard qui les met en relation avec le présent. D'où la possibilité de rapprocher cette dialectique de l'image avec celle qu'énonce Deleuze entre l'image actuelle et l'image virtuelle, puisque l'image actuelle est une image qui se manifeste au Maintenant, tandis que l'image virtuelle est une trace du passé, d'Autrefois, supportée par la mémoire. Et l'éclair qui conjugue le passé avec le présent s'apparente à ce que Deleuze nomme cristal de temps.

Dans un ouvrage où il s'intéresse justement à la temporalité de l'image, Didi-Huberman résume les positions de Deleuze et Benjamin: "L'image est d'abord un cristal de temps, la forme construite et flamboyante tout à la fois, d'un choc fulgurant où L'Autrefois rencontre le Maintenant dans un éclair pour former une constellation/13. ». Les enjeux théoriques qu'une notion comme le cristal de temps soulève sont d'une grande importance, car c'est lui qui nous permet d'expérimenter le temps. Les circuits qu'il forme entre l'image actuelle et son image virtuelle produisent un relief dans le temps, et le rendent perceptible. Autrement dit, ils forment une perspective dans le temps. Tout aussi passionnant que puisse être le
/12 Walter Benjamin, Le Livre des passages, trad. J.Lacoste, Paris, Cerf, 1989, p. 478-479.

/13 Georges Didi-Huberman, Devant le temps, Paris, Minuit, 2000, p. 241. 
/14 Gilles Deleuze, op. cit., p. 109.

/15 ibid., p. 358.

/16 Georges Didi-HuberMan, L'Image survivante, Paris, Minuit, 2002, p. 320. rapprochement avec le cristal pour penser la temporalité de l'image, du fait qu'il met en relation une forme et une matière, il est néanmoins problématique de penser que sa cristallisation soit à proprement parler l'effet du temps, comme le suggèrent ces deux passages de L'Image-temps: «Ce qui constitue l'image-cristal, c'est l'opération la plus fondamentale du temps: puisque le passé ne se constitue pas après le présent qu'il a été, mais en même temps, il faut que le temps se dédouble à chaque instant en présent et passé, qui diffèrent l'un de l'autre en nature/14. » «[...] Ce que nous voyons dans le cristal, ce n'est plus le cours empirique du temps comme succession de présents, ni sa représentation indirecte comme intervalle ou comme tout, c'est sa présentation directe, son dédoublement constitutif en présent qui passe et passé qui se conserve, la stricte contemporanéité du présent avec le passé qu'il sera, du passé avec le présent qu'il a été/15. ". II ne fait aucun doute que, pour Deleuze, le temps se dédouble. C'est même l'élément constitutif du temps. La cristallisation est l'opération du temps, comme si ce dernier était responsable de son propre dédoublement. Didi-Huberman écrit d'ailleurs dans L'image survivante que «le temps ne fait pas que s'écouler, il travaille/16 ». Le problème de ces remarques, c'est qu'en voulant que le temps se dédouble de lui-même, elles lui attribuent une valeur active, participative.

Longtemps on a pensé que le temps s'écoulait à une vitesse stable, de manière linéaire, et qu'il constituait le cadre à l'intérieur duquel nos actions se déployaient. Telle était du moins la conception moderne du temps. Cela dit, ce modèle a été profondément ébranlé au siècle dernier lorsqu'Albert Einstein et Hermann Minkowski ont développé, à partir des mathématiques, les notions de relativité et d'espace-temps. De fait, en démontrant qu'il est possible d'altérer l'écoulement du temps en fonction de la vitesse à laquelle un observateur se déplace, ils ont permis de comprendre que le temps est relatif à l'interaction entre les parties qui forment l'Univers: il n'est pas un cadre à l'intérieur duquel celles-ci évoluent, pas plus qu'il n'est responsable de cette interaction. Comment dès lors expliquer que le temps puisse être à l'origine de sa propre perspective, qu'il puisse se préfigurer lui-même?

On doit se poser la question suivante : est-ce le temps qui se dédouble, ou quelque chose à même notre manière d'être qui opère ce dédoublement? Si quelque chose en nous opère ce dédoublement et rend contemporain par là-même le passé à travers notre perception du présent, alors la cristallisation du temps, ou la constellation de l'image, ne doit pas être pensée du côté de l'image en soi, mais à même notre relation subjective à celle-ci. Et un film comme L'Arche russe appuie cette idée précisément parce qu'il situe la tension temporelle au cœur 
de la relation entre un donnant à voir et ce qui est donné à voir, c'està-dire entre le présent du regard, et les traces du passé qui s'actualise à travers lui.

\section{Interpréter le présent, faire face à l'Histoire}

À en croire L'Arche russe, entrer dans le Musée de l'Ermitage c'est s'aventurer dans un lieu de contemporanéité, soit un espace où les diverses strates de l'histoire se superposent à même le regard, et où celles-ci ne sont jamais dépassées. Martin Heidegger écrit en ce sens qu'il « est impossible d'échapper à l'histoire. Ici, l'histoire désigne le passé, mais un passé qui ne continue pas moins d'exercer ses effets/17 », et ces effets se font sentir - comme c'est le cas dans L'Arche russe - à même le regard du narrateur. Comme quoi il ne peut y avoir de regard purement actuel ou entièrement détaché du passé.

En rendant tangible l'enracinement du présent dans le passé, et ce à même la composition de l'image, l'œuvre de Sokourov rend palpable le fait que notre propre perspective sur le monde se fonde sur notre héritage culturel. Paraphrasant Heidegger, Alain Boutot écrit que «le Dasein, soit l'être-là, est toujours jeté dans un monde, livré ou remis à un là dont il n'est pas lui-même l'origine et qui pourtant constitue son être/18 ». Ce qui revient à dire que l'héritage culturel qui nous précède, c'est-à-dire ce qui s'actualise à travers l'image, façonne non seulement ce qui est perçu par le regard, mais la composition même du regard. Et toute la vie subjective se joue dans cet intervalle.

L'Arche russe se déroule d'ailleurs comme l'évolution d'une subjectivité. Si le plan final évoque la mort, la séquence d'ouverture correspond quant à elle à une naissance. Elle débute dans l'obscurité et, lorsque la lumière se fait, un regard surgit - comme s'il venait au jour - au milieu d'une scène complexe. Jeté dans le monde, ce regard doit opérer un travail d'interprétation à même l'image; pour reprendre la terminologie de Deleuze, des images virtuelles doivent se cristalliser à l'image actuelle.

C'est pourquoi, si l'on revient à la question posée précédemment, à savoir de quelle nature est le dédoublement du temps, tout porte à croire que c'est du côté de l'interprétant qu'il faut chercher. Car ce sont précisément des repères encyclopédiques/19 qui, en jouant le rôle d'interprétants, assurent le rapprochement entre l'histoire qui précède - et détermine - l'actualité de l'image.

Il importe ici d'évoquer la théorie sémiotique de Charles S. Peirce car, en faisant de l'interprétation une opération logique, elle permet de penser le mouvement par lequel le donnant à voir et le donné à voir, ainsi que le présent et son passé, s'agglutinent. Quand Peirce
/17 Martin Heidegger, Etre et temps, trad. E. Martineau, édition numérique hors-commerce, 1985, p. 286, en ligne [t.p.m.p.free.fr/textes/ Heidegger_etre_et_ temps.pdf].

/18 Martin Heidegger, Ontologie. Herméneutique de la factivité, trad. A. Boutot, Paris, Gallimard, 2012, p. 14 .

/19 Quant à l'utilisation de la notion d'« encyclopédie ", je me réfère aux textes d'Umberto Eco Lector in fabula. Le rôle du lecteur ou la Coopération interprétative dans les textes narratifs, trad. M. Bouzaher, Paris, Grasset, 1985 ; Sémiotique et philosophie du langage, trad. M. Bouzaher, Paris, Presses Universitaires de France, 1988. 
/ 20 Jean-Pierre Luminet, L'Univers chiffonné, Paris, Fayard, 2005, p. 70.

introduit la notion d'« interprétant » dans sa théorie de la sémiose, il élabore un modèle qui tient compte de l'héritage culturel, sur le mode de l'habitude et de la croyance, qui est déterminant dans tout processus de signification. Et grâce à cette notion, on peut penser que c'est à l'intérieur même des processus sémiotiques que le dédoublement du temps s'opère, précisément parce que ceux-ci actualisent des signes perçus par le passé dans une perception présente et forment, comme l'éclair de Benjamin, une constellation qui donne à voir tout à la fois le présent et le passé.

C'est donc dire que le dédoublement du temps, soit la rencontre entre le passé et le présent, s'effectue au sein même de l'imaginaire puisque la sémiose mobilise des interprétants qui appartiennent à diverses couches historiques. C'est d'ailleurs ainsi qu'on peut comprendre pourquoi le passé n'est jamais complètement dépassé, du moins pas tant et aussi longtemps que ses interprétants peuvent s'actualiser à nouveau.

Quant à L'Arche russe, on peut dès lors affirmer qu'il met en scène l'historicité de l'imaginaire et ce, parce qu'il illustre la dialectique qu'il y a entre la présence d'un regard et l'histoire qui le traverse. Autrement dit, la traversée des deux cents ans de l'histoire de l'Ermitage qui hantent le musée ne rend pas seulement compte de l'évolution des formes de représentation qu'a connue l'histoire de l'art, mais cette visite extraordinaire du musée réalise une poétisation des processus sémiotiques par lesquels le présent s'enracine dans le passé et le passé s'actualise dans le présent.

De ces processus on peut effectivement dire, avec Deleuze, que des cristaux de temps émergent. C'est dans l'agencement de ces cristaux que des bribes d'histoire surgissent dans L'Arche russe: des constellations de sens qui éclairent tout à la fois l'Autrefois et le Maintenant. Par ailleurs, le rapprochement à la constellation n'est pas dépourvu d'intérêt pour exemplifier l'image hétérogène qu'on se fait du temps si l'on considère que lorsque les astrophysiciens observent l'Univers, c'est-à-dire qu'ils étudient le mouvement des astres qui forment les constellations, ils sont eux-mêmes incapables de prouver l'homogénéité du temps: "L'homogénéité, en revanche, peut sembler une hypothèse moins réaliste. En premier lieu, pour se rendre compte de l'homogénéité de l'espace, il faut pouvoir bouger instantanément dans l'Univers, ce qui est impossible. L'observateur immobile regarde l'Univers à des époques différentes du passé, il ne peut donc pas en prouver expérimentalement l'homogénéité/20. ».

De la même manière qu'on ne peut observer l'Univers qu'à des époques différentes du passé, un film comme L'Arche russe illustre comment notre rapport au monde est tout aussi inactuel. Ce n'est jamais le 
présent en soi qui est observé, car des figures appartenant au passé participent constamment, en tant qu'interprétants du présent, à notre activité sémiotique, de manière à ce que l'imaginaire, à l'instar du film de Sokourov, apparaît comme une sorte de kaléidoscope temporel.

Cette logique désarticule évidemment la conception traditionnelle du temps qui, en tant qu'il est pensé comme successif, ne suffit plus car il doit également être conçu selon une certaine juxtaposition, voire même une superposition. Toutes les strates de l'histoire sont mises en relation à l'intérieur d'un même cadre, comme s'il y avait une certaine contemporanéité dans l'image. Dans un article publié précédemment/21, je défendais justement l'idée qu'il y a contemporanéité au sein de l'imaginaire, c'est-à-dire qu'il y a toujours plus d'un temps qui se rencontrent en même temps pour penser un même temps: le présent.

À l'inverse des récits qui, selon Paul Ricœur, « nous aident à configurer notre expérience temporelle confuse, informe, à la limite muette/22 », l'image cinématographique de L'Arche russe est précisément au service de la nature confuse et informe de notre expérience du temps. Comme ce film met en évidence l'inactualité du regard vécu, on peut affirmer que sa tension temporelle se rapporte directement à notre expérience intime du temps.

Par conséquent, si l'hypothèse que Ricœur défend dans Temps et récit veut que « toute configuration narrative s'achève dans une refiguration de l'expérience temporelle/23 ", l'analyse permet de penser que c'est précisément une configuration sémiotique, une interprétation fondée sur des traces du passé, qui rend possible l'expérience même du temps. Autrement dit, notre horizon temporel s'ouvre au cœur de la béance entre un donnant à voir et un donné à voir/24.

Au bout du compte, si Bresson écrit dans ses Notes sur le cinématographe qu'un réalisateur doit faire apparaître ce qui sans lui ne serait peut-être jamais vu/25, Sokourov donne certainement à voir la confusion temporelle dans laquelle on se trouve et ce, en faisant du musée un espace de contemporanéité. Un rapprochement peut donc être fait entre le musée et l'imaginaire, rapprochement qui pourrait être consolidé dans un travail futur par le truchement de la notion d' « encyclopédie », car l'exploration de l'un devient une métaphore pour découvrir les logiques de l'autre. Si le film de Sokourov rend ce parallèle visible, c'est précisément parce que la caméra subjective agit tel un regard vécu et que l'image qu'elle donne à voir est, en ce sens, le produit d'un imaginaire. Un imaginaire qui, par surcroit, est dynamique dû au fait qu'il est en mouvement.
/21 Francis Gauvin, «Pour une contemporanéité de l'imaginaire", dans Salon double, [http://salondouble. contemporain.info/ antichambre/pourune-contemporan-itde-l-imaginaire], page consultée le 10 octobre 2013.

/22 Paul Ricœur, Du texte à l'action. Essais d'herméneutique II, Paris, Seuil, 1986, p. 20.

/23 Paul Ricœur, Temps et récit 3. Le temps raconté, Paris, Seuil, 1991, p. 9.

/ 24 II resterait à questionner les modalités de cette interprétation du temps, comment elle cristallise une histoire au sein d'une image, mais je ne peux pas m'avancer davantage en ce sens dans le cadre de cette analyse de L'Arche russe. Ces questions seront néanmoins centrales à la thèse de doctorat que je prépare.

/25 « Fais apparaitre ce qui sans toi ne serait, peut-être jamais vu. » Robert Bresson, Notes sur le cinématographe, Paris, Gallimard, 1988, p. 82.

\section{Francis Gauvin}

